\title{
EXISTENCE AND NONEXISTENCE \\ OF LEAST ENERGY NODAL SOLUTIONS \\ FOR A CLASS OF ELLIPTIC PROBLEM IN $\mathbb{R}^{2}$
}

\author{
Claudianor O. Alves - Denilson S. Pereira
}

\begin{abstract}
In this work, we prove the existence of least energy nodal solutions for a class of elliptic problem in both cases, bounded and unbounded domain, when the nonlinearity has exponential critical growth in $\mathbb{R}^{2}$. Moreover, we also prove a nonexistence result of least energy nodal solution for the autonomous case in whole $\mathbb{R}^{2}$.
\end{abstract}

\section{Introduction}

This paper concerns with the existence of least energy nodal solutions for the following class of elliptic problem

$$
\left\{\begin{array}{l}
-\Delta u+V(x) u=f(u) \quad \text { in } \Omega, \\
u \in H_{0}^{1}(\Omega),
\end{array}\right.
$$

where $\Omega \subset \mathbb{R}^{2}$ is a smooth bounded domain or $\Omega=\mathbb{R}^{2}, V: \bar{\Omega} \rightarrow \mathbb{R}$ is a continuous function verifying some hypotheses which will be fix later on. Concerning the nonlinearity, we assume that $f: \mathbb{R} \rightarrow \mathbb{R}$ is a $C^{1}$-function, which can have an exponential critical growth at both $+\infty$ and $-\infty$, that is, it behaves like $e^{\alpha_{0} s^{2}}$,

2010 Mathematics Subject Classification. 35A15, 35J15.

Key words and phrases. Variational methods, exponential critical growth, nodal solution. Partially supported by CNPq - Grant 304036/2013-7. 
as $|s| \rightarrow \infty$, for some $\alpha_{0}>0$. More precisely,

$$
\begin{array}{ll}
\lim _{|s| \rightarrow \infty} \frac{f(s)}{e^{\alpha|s|^{2}}}=0 & \text { for all } \alpha>\alpha_{0}, \\
\lim _{|s| \rightarrow \infty} \frac{f(s)}{e^{\alpha|s|^{2}}}=\infty & \text { for all } \alpha<\alpha_{0},
\end{array}
$$

(see [20]). In the last years, we have observed that the existence of nodal solution has received a special attention of a lot of researches. In Cerami, Solimini and Struwe [19], the authors showed the existence of multiple nodal solutions for the following class of elliptic problem with critical growth

$$
\begin{cases}-\Delta u-\lambda u=|u|^{2^{*}-2} u & \text { in } \Omega, \\ u=0 & \text { on } \partial \Omega,\end{cases}
$$

where $\Omega=B_{R}(0) \subset \mathbb{R}^{N}, N \geq 7,2^{*}=2 N /(N-2)$ and $\lambda \in\left[0, \lambda_{1}\right]$, with $\lambda_{1}$ being the first eigenvalue of $\left(-\Delta, H_{0}^{1}(\Omega)\right)$. In Bartsch and Willem [12], the existence of infinitely many radial nodal solutions was proved for the problem

$$
\left\{\begin{array}{l}
-\Delta u+u=f(|x|, u) \quad \text { in } \mathbb{R}^{N}, \\
u \in H^{1}\left(\mathbb{R}^{N}\right),
\end{array}\right.
$$

where $f$ is a continuous function with subcritical growth and verifying some hypotheses. In Cao and Noussair [17], the authors studied the existence and multiplicity of positive and nodal solutions for the following class of problems

$$
\left\{\begin{array}{l}
-\Delta u+u=Q(x)|u|^{p-2} u \quad \text { in } \mathbb{R}^{N}, \\
u \in H^{1}\left(\mathbb{R}^{N}\right)
\end{array}\right.
$$

by supposing $2<p<(N+2) /(N-2), N \geq 3$ and some technical conditions on $Q$. In that paper, the main result connects the number of positive and nodal solutions with the number of maximum points of function $Q$.

In Castro, Cossio and Neuberger [18] and Bartsch and Wang [13], the authors studied the existence of nodal solution for a problem like

$$
\begin{cases}-\Delta u=f(u) & \text { in } \Omega, \\ u=0 & \text { on } \partial \Omega,\end{cases}
$$

where $\Omega$ is a smooth bounded domain and $f$ verifies some hypotheses. In [18], it was assumed that $f$ is superlinear, while in [10] that $f$ is asymptotically linear at infinity. In Bartsch and Weth [10], existence of multiple nodal solutions was also considered for problem $\left(\mathrm{P}_{3}\right)$. 